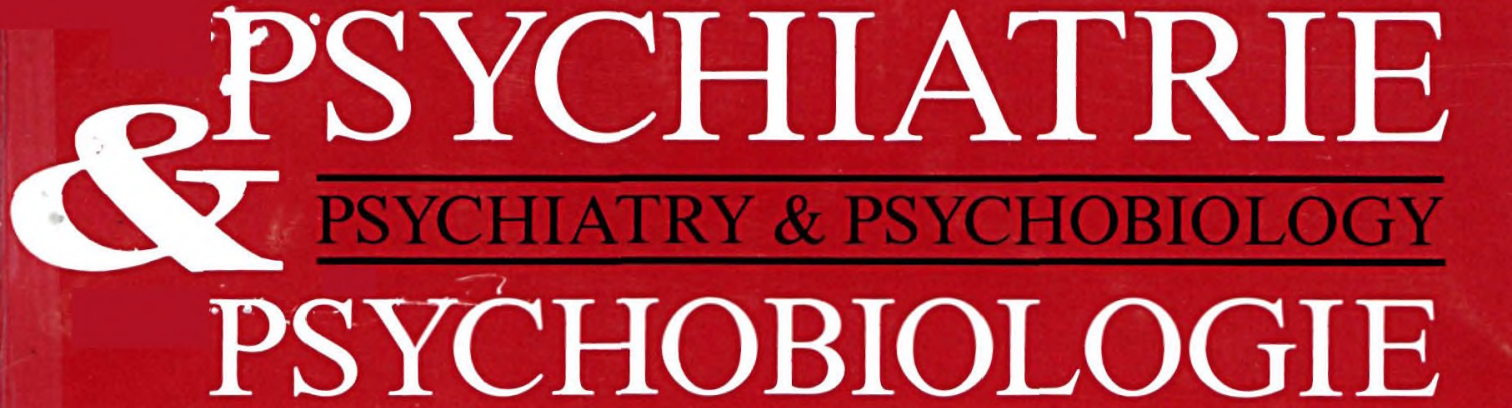

VOL $5 / \mathrm{N}^{\circ} 4$

ISSN 0767-399 X

SCSPED II (1) 231-282

PSYPEK (5) (4)

Rédacteurs en chef: P Boyer, Paris/Sainte-Anne, JD Guelfi, Paris/Sainte-Anne, Y Lecrubier, Paris/Salpêtrière

\section{Rédacteurs associés}

Psychopathologie

J AdEs, Paris/Colombes

HS AKISKAL, Memphis, TN

J DALERY, Lyon

R Klein-Gittelman, New York

H Katschnig, Vienne

R LEVY, Londres

HG POPE, Belmont, MA

W REIN, Tübingen

Diagnostic et classification

N Mombour, Munich

CB Pull, Luxembourg

N SArtorius, Genève

RL SpITZER, New York

\section{Psychobiologie}

M ACKenHeIL, Munich

\section{Comité Scientifique}

NC Andreasen, lowa City, IO

M Asberg, Stockholm

JL Ayuso, Madrid

TA BAN, Nashville, TN

P Berner, Vienne

D Bobon, Liège

M Bourgeors, Bordeat

M CASAS, Barcelone

J Cottraux, Lyon

R DANTzER, Bordeaux
P Baumann, Lausanne

JP Boulenger, Caen

D KeMALı, Naples

P LEMOINE, Lyon/Bron

Psychothérapie

A Braconnier, Paris

S Consolı, Paris

P Jeammet, Paris

C Perris, Ümea

I MARKS, Londres

Chimiothérapie

G Cassano, Pise

JA Costa E Silva, Rio de Janeiro

D Ginestet, Paris/Villejuif

G Sedvall, Stockholm

R TAKahashi, Tokyo +

A Villeneuve, Beaufort/Québec

H Dufour, Marseille

A Feline, Paris/Kremlin-Bicêtre

J Glowinski, Paris

C Kordon, Paris

S Lebovicl, Paris

M Le MoAl, Bordeaux

-.......... Doric/rolomhes
Psychopharmacologie clinique

JM DANION, Strasbourg

SZ Langer, Paris

AJ PUECh, Paris

TW RobBins, Cambridge, UK

Comportement animal et pharmacologie

J Costentin, Rouen

Ph Soubrie, Montpellier

Neuropsychologie

$M$ de Bonis, Paris/Kremlin-Bicêtre

JA Gray, Londres

Epidémiologie et Analyse des données

J Fermanian, Paris

$\mathrm{R}$ von FRENCKELL, Liège

J Lellouch, Paris/Villejuif

JP LÉPINE, Paris

F Roullon, Paris/Colombes
J Mendlewicz, Bruxelles

J Pellet, Saint-Etienne

P Ріснот, Paris

D SCHAlling, Stockholm

JC Schwartz, Paris

P SIMON, Paris

L Singer, Strasbourg

D Widlocher, Paris

E Zarifian, Caen

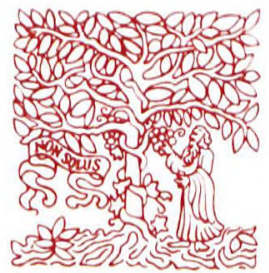



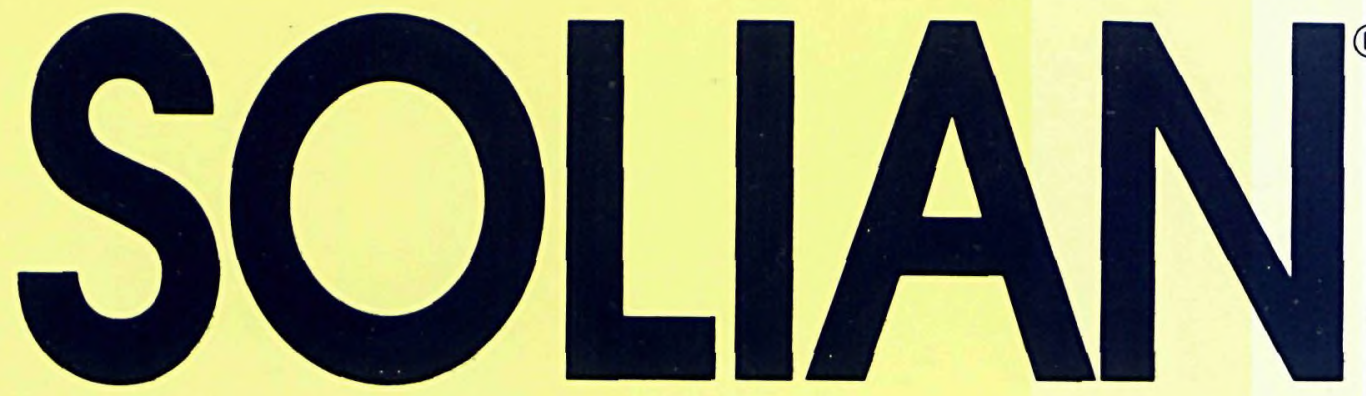

(R)
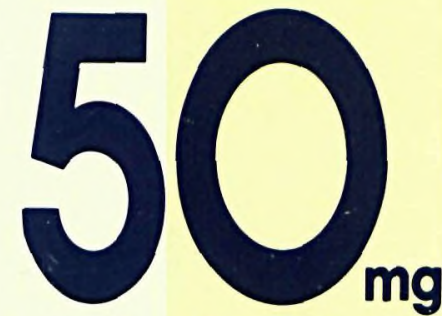

\section{amisulpride}

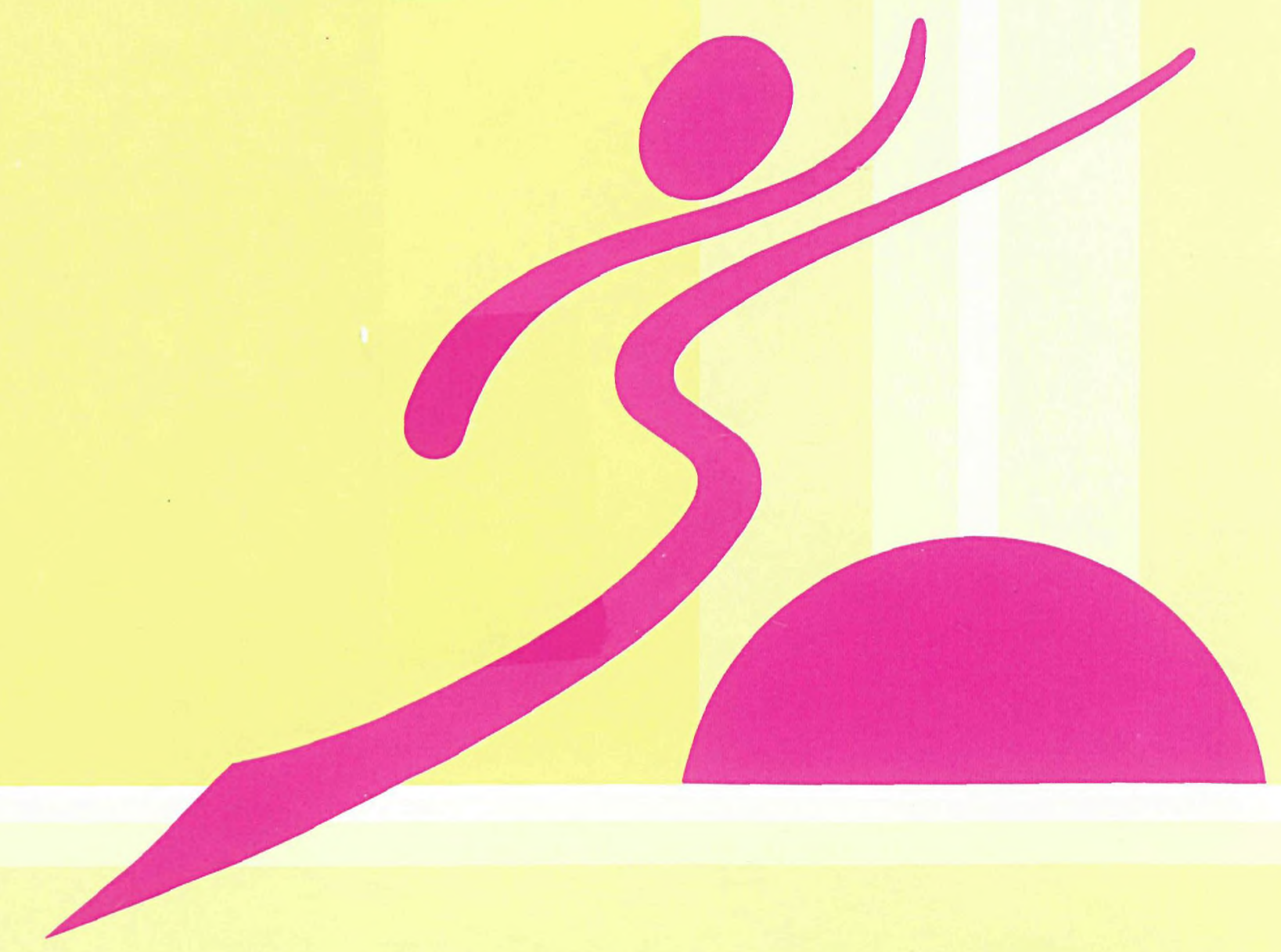

ANTIDÉFICITAIRE RAPIDE ET MANIABLE

PROPRIÉTES, Neuroleptique caractérisé par sa rapidité d'action, antiproductif ou antidéficitaire selon la dose utilisée - SORT DU MÉDICAMENT , Se reporter au Vidal - INDICATIONS, Traitement des psychoses - Etats déficitaires des schizophrénies, des evvolutions psychotiques résiduelles, des états d'inhibition avec ralentissement - POSOLOGIE, La forme Solian $50 \mathrm{mg}$ est particulièrement adaptée au traitement des états déficitaires et états d'inhibition, I a 5 comprimés par jour (coatj.t. . 2.31 a ll.55 F). Le traitement des psychoses productives se fera plutot a l'aide de Solian $200 \mathrm{mg}$ ou de Solian injectable - MISE EN GARDE, Suspendre le traitement en cas d'hyperthermie, surtout s'il est utilise d forte dose, cette hyperthermie peut être l'un des éléments du syndrome malin des neuroleptiques - EFFETS INDÉSIRABLES, Sédation ou somnolence (a forte dose), dyskinésies précoces (torticolis spasmodique, crises oculogyres, trismus) cédant d un antiparkinsonien anticholinergique, syndrome extrapyramidal cédant partiellement aux antiparkinsoniens anticholinergiques, dyskinessies tardives qui pourraient etre observées comme avec tous les neuroleptiques au cours de cures prolongées, hyotension modérée (à forte dose). impuissance, frigidité, aménorrhée, galactorrhée, gynécomastie, hyperprolactinémie, prise de poids - PRÉCAUTIONS D'EMPLOI, Déconseiller l'absorption d'alcool pendant le traitement. Réduire la posologie et prescrire des cures discontinues chez linsuffisant renal grave. Renforcer la surveillance chez les épileptiques et les sujets suspects d'etre porteurs d'un phéochromocytome. Prudence chez le sujet age, le parkinsonien, la femme enceinte (par prudence, ne pas utiliser au cours du premier trimestre de grossesse)- VIGILANCE EI CONDUITE, Risque de somnolence-INTERACIIONS , Potentialisation possible des hypotenseurs, des antihypertenseurs et des dépresseurs du système nerveux central. Association da la lévodopa déconseillée - SURDOSAGE, Syndrome parkinsonien gravissime, coma, traitement symptomatique - PRÉSENTATIONS, Comprimés dosés a $50 \mathrm{mg}$ d'amisulpride - boîte de 30 - A.M.M. 328 445.2 - TABLEAU A - PRIX PUBLIC, Comprimés a 50 mg , 69.30 F + S.H.P. - Remboursé par la Sécurité Sociale d $70 \%$. Agrée aux collectivités. 


\section{PSYCHIATRIE \& PSYCHOBIOLOGIE PSYCHIATRY \& PSYCHOBIOLOGY}

\section{OBJECTIFS}

L'objectif de Psychiatrie \& Psychobiologie est de publier les résultats de travaux originaux dans les domaines qui intéressent, à l'heure actuelle, la Psychiatrie tels que: psychopathologie, nosographie, chimiothérapie, psychothérapie, méthodologie des essais cliniques, perturbations biologiques et pathologie mentale, psychophysiologie, neuropsychologie, comportement animal.

Cet objectif large mais centré sur la publication d'articles originaux est destiné à:

1) faciliter la circulation des idées et des travaux au sein des pays européens,

2) permettre une meilleure connaissance mutuelle des évolutions survenant dans la communauté psychiatrique internationale.

Les communications brèves seront publiées dans des délais rapides afin de rendre le plus actuel possible les informations présentées dans cette revue.

\section{AIMS AND SCOPE}

Psychiatry \& Psychobiology presents the results of original research relative to those domains which are presently of interest to psychiatry: psychopathology, nosography, chemotherapy, psychotherapy, clinical methodology, biological disorders and mental pathology, psychophysiology, neuropsychology, as well as animal behavior.

This large scope, emphasizing, nevertheless, the publication of original articles, is meant to:

1) encourage the exchange of ideas and research within Europe,

2) enable, within the international psychiatric community, an improved level of scientific communication.

Short communications will be published rapidly in order to keep information in this journal up-to-date.

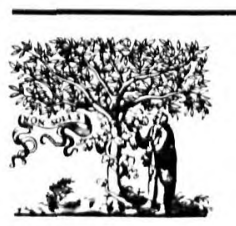

\section{ÉDITIONS SCIENTIFIQUES ELSEVIER}

29, rue Buffon, F-75005 Paris

Tél: (1) 47071122 - Télex: 202400 F - Télécopie: (1) 43368093

\section{ABONNEMENTS/SUBSCRIPTIONS 1990 (Vol 5)}

Un an/annual subscription; 6 numéros/6 issues: FF 490 (France), FF 540 Autres pays (other countries).

Adresser ordre et paiement à /Address order and payment to: Éditions Scientifiques Elsevier.

Banque/Bank: CCP Paris, $N^{\circ} 30041000011904540 \mathrm{H} 020 / 70$.

- Les abonnements sont mis en service dans un délai de 4 semaines après réception du paiement. Ils partent du premier numéro de l'année/Subscriptions begin 4 weeks after receipt of payment and start with the first issue of the calendar year.

- Les numéros de l'année et les volumes antérieurs doivent être commandés à l'éditeur/Back issues and volumes should be ordered from the publisher. - Les réclamations pour les numéros non reçus doivent parvenir dans un délai maximal de 3 mois après la parution/Claims for missing issues should be made within three months of publication.

- Les revues sont expédiées par voie aérienne sans supplément/Journals are sent by air delivery.

PUBLICITÉ/ADVERTISING: Éditions Scientifiques Elsevier (Chantal Libert).

\section{(C) 1990 Éditions Scientifiques Elsevier, Paris}

Tous droits de traduction, d'adaptation et de reproduction par tous procédés réservés pour tous pays. Loi du 11 mars 1957 . Il est interdit de reproduire, mẻme partiellement, la présente publication sans l'autorisation de l'éditeur ou du Centre Français du Copyright, 6 bis, rue Gabriel-Laumain, 75010 Paris.

All rights reserved. No part of this publication may be translated, reproduced, stored in a retrieval system or transmitted in any form or by any other means, electronic, mechanical, photocopying, recording or otherwise, without prior permission of the publisher.

Imprimé en France par l'Imprimerie Saint-Paul, 55000 Bar-le-Duc

Dépôt légal: octobre 1990. N $9-90-898$

Le directeur de la publication: Alain Devanlay Commission paritaire: 68321 Périodicité: 6 numéros/an 


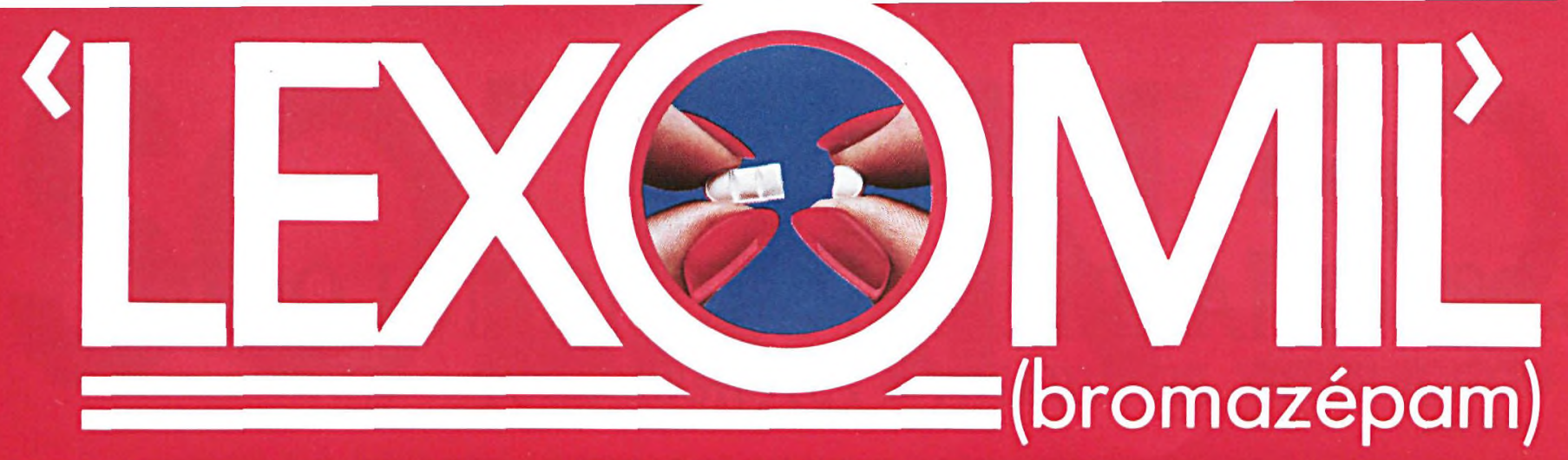

\section{LÈVE LINHIBITION ANXIEUSE}

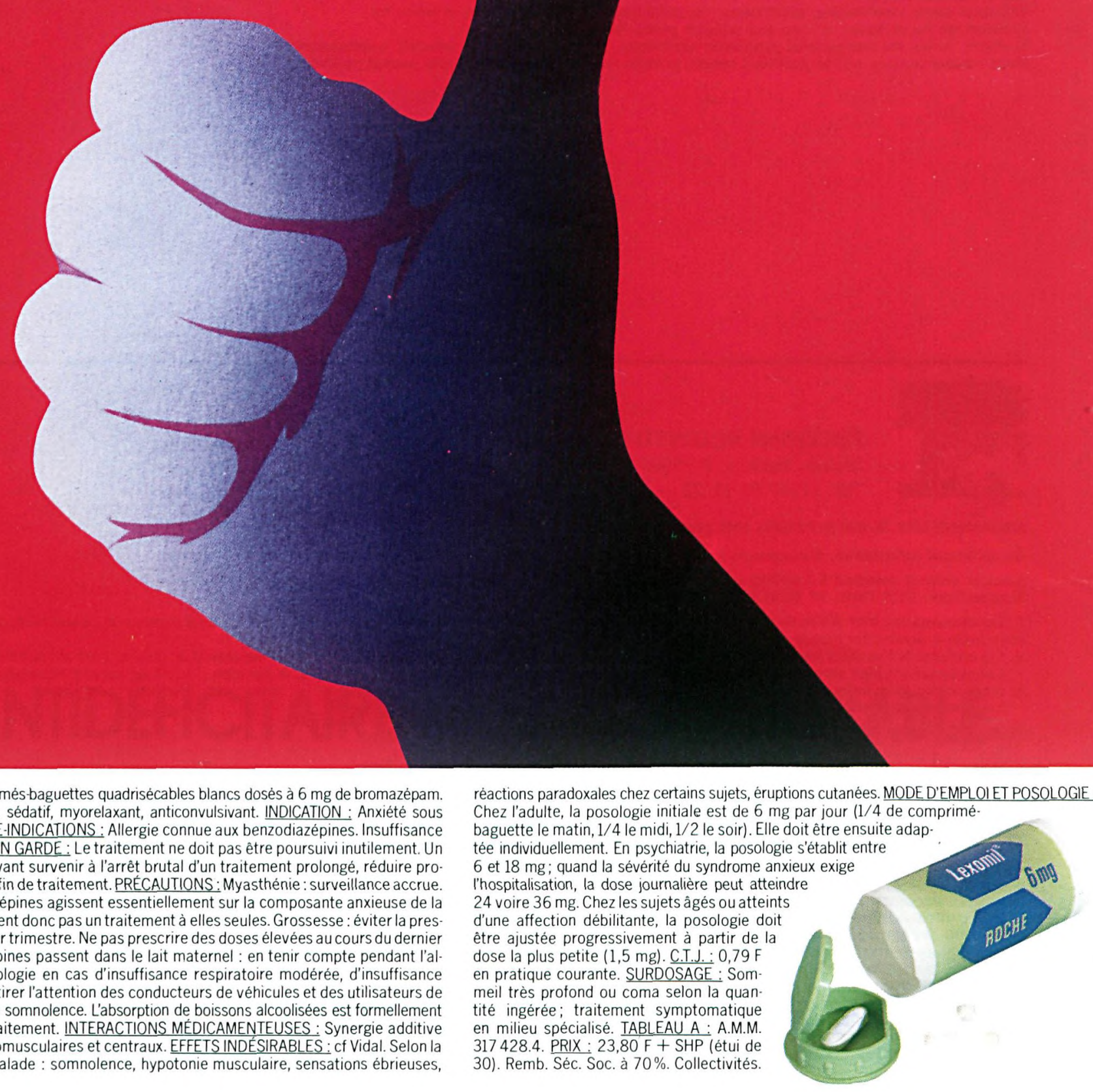

PRESENTATION : 30 comprimés-baguettes quadrisécables blancs dosés à $6 \mathrm{mg}$ de bromazépam. PROPRIÉTÉS: Anxiolytique, sédatif, myorelaxant, anticonvulsivant. INDICATION: Anxiété sous toutes ses formes. CONTRE-INDICATIONS: Allergie connue aux benzodiazépines. Insuffisance respiratoire sévère. MISE EN GARDE : Le traitement ne doit pas être poursuivi inutilement. Un syndrome de sevrage pouvant survenir à l'arrêt brutal d'un traitement prolongé, réduire pro gressivement les doses en fin de traitement. PRÉCAUTIONS: Myasthénie: surveillance accrue. Dépression : les benzodiazépines agissent essentiellement sur la composante anxieuse de la dépression et n'en constituent donc pas un traitement à elles seules. Grossesse : éviter la pres cription au cours du premier trimestre. Ne pas prescrire des doses élevées au cours du dernie trimestre. Les benzodiazépines passent dans le lait maternel : en tenir compte pendant l'allaitement. Adapter la posologie en cas d'insuffisance respiratoire modérée, d'insuffisance rénale et/ou hépatique. Attirer l'attention des conducteurs de véhicules et des utilisateurs de machines sur les risques de somnolence. L'absorption de boissons alcoolisées est formellement déconseillée pendant le traitement. INTERACTIONS MÉDICAMENTEUSES : Synergie additive avec les dépresseurs neuromusculaires et centraux. EFFETS INDÉSIRABLES: cf Vidal. Selon la dose et la sensibilité du malade : somnolence, hypotonie musculaire, sensations ébrieuses, réactions paradoxales chez certains sujets, éruptions cutanees.MODE D'EMPLOIET POSOLOGIE : Chez l'adulte, la posologie initiale est de $6 \mathrm{mg}$ par jour (1/4 de comprimé baguette le matin, $1 / 4$ le midi, $1 / 2$ le soir). Elle doit être ensuite adaptée individuellement. En psychiatrie, la posologie s'établit entre 6 et $18 \mathrm{mg}$; quand la sévérité du syndrome anxieux exige l'hospitalisation, la dose journalière peut atteindre 24 voire $36 \mathrm{mg}$. Chez les sujets âgés ou atteints d'une affection débilitante, la posologie doi etre ajustée progressivement à partir de . en pratique courante. SURDOSAGE: Som meil très profond ou coma selon la quaningérée: traitement symptomatique en milieu spécialisé. TABLEAU A: A.M.M. 8.4. PRIX: $23,80 \mathrm{~F}+$ SHP (étuide 
Psychiatr \& Psychobiol 5 (1990), $N^{\circ} 4$

Central $\mathrm{D}_{2}$ receptor blockade and antipsychotic effects of neuroleptics. Preliminary study with positron emission tomography

JL Martinot, ML Paillère-Martinot, C Loc'h, P Péron Magnan, B Mazoyer, Y Lecrubier, P Hardy,

B Beaufils, JF Allilaire, B Mazière, MF Slama, A Syrota (Paris, Villejuif, France) ..........

Troubles du raisonnement et schizophrénie: une revue critique

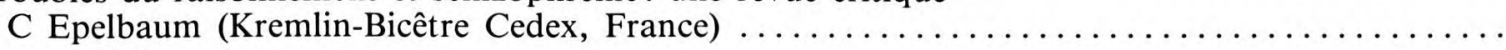

Psychopathological and sociodemographic characteristics of 1231 depressed patients with and without co-existing alcoholism

H Lôo, W Rein, A Souche, H Dufour, JD Guelfi, R Malka, JP Olié (Paris, France; Lausanne,

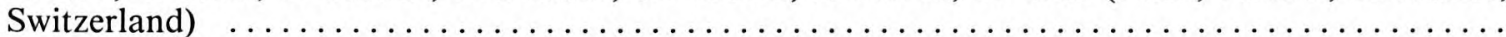

Intérêts et limites du test de Folstein dans l'évaluation des performances de sujets âgés, non déments et déments, hospitalisés

$\mathrm{X}$ Hoff (Paris, France)

Prediction of serum sodium based on diurnal weight gain among schizophrenics subject to water intoxication

WVR Vieweg, DP Harrington, RA Leadbetter, PL Hundley, GR Yank (Virginia, USA) .....

Typologie de patients anxieux et validation de l'échelle Fard

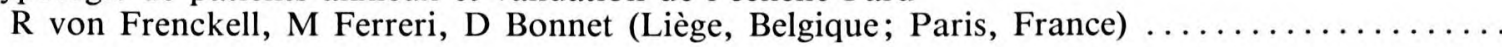

Dans la littérature

Agenda

Recommandations aux auteurs

Instructions to authors

Cité dans: Biological Abstracts; CNRS/Pascal; Excerpta Medica; Psychological Abstracts 


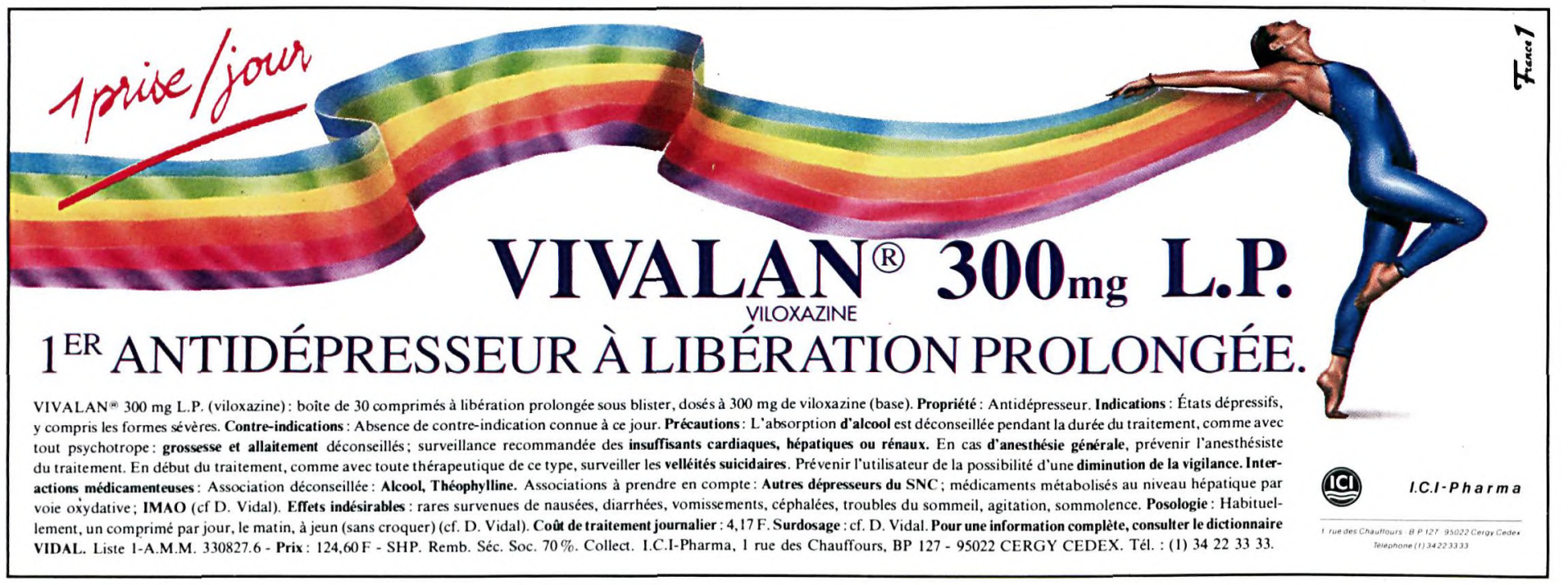

